Methods: Soluble ES and MMP-9,were measured in 68 patients with KD,20 patients with febrile disease and 20 healthy children by using double antibody sandwich enzyme linked immunosorbent assay (ELISA). Patients with KD were separated into acute phase group, subacute phase group, recovery phase group. coronary artery lesion group (CAL), non-coronary artery lesion group (NCAL). Results: KS and MMP-9 levels in the acute phase group,subacute phase group were significantly higher than those in the healthy group. Plasma ES and MMP-9 levels of CAL group were significanfly higher than those of NCAL group in acute phase. ne peak level of ES and MMP-9 appeared in the acute phase.There Was a significant correlation between KS and MMP-9 levels in KD patients $(r=0.643$, $\mathrm{P}<0.01)$.

Conclusions: the increase of plasma PS and ES levels in KD acute phase and subacute phase might play an important role in the pathophysiology of the VasCUlax damage.KS and MMP-9 may potentially be a predictor of CAL in patients.

Disclosure of Interest: None declared

DOI: 10.1136/annrheumdis-2017-eular.2123

\section{AB0570 CLASSIFICATION, EPIDEMIOLOGY, AND CLINICAL PHENOTYPES OF PRIMARY VASCULITIDES IN COLOMBIA}

J.E. Barahona-Correa $^{1,2}$, Ó.E. Gómez-Cárdenas ${ }^{3}$, J.C. Sarmiento Monroy ${ }^{1,2}$, R.D. Mantilla ${ }^{1,2} .{ }^{1}$ Rheumatology, Center of Dermatology and Rheumatology (FUNINDERMA); ${ }^{2}$ Center for Autoimmune Diseases Research (CREA), Universidad del Rosario; ${ }^{3}$ Department of physiological sciences, School of Medicine, Pontifical Xavierian University, Bogotá, Colombia

Background: Primary vasculitides usually pose a diagnostic challenge. They represent a wide spectrum of heterogeneous vascular disorders characterized by variable target vessel involvement, vascular abnormalities, and end organ damage [1]. Several studies about primary vasculitides expose epidemiologic data, mainly from North America and Europe. Nevertheless, data in Latin America is scarce [2]. Objectives: To describe the prevalence and clinical presentation of primary vasculitides.

Methods: This was an observational cross-sectional study in which wellcharacterized patients were assessed from 2001 to 2016. Each patient was evaluated by the same rheumatologist in a single rheumatology outpatient center in Bogota, Colombia. Patients were classified either according to ACR 1990 criteria (Granulomatosis with polyangiitis, Eosinophilic granulomatosis with polyangiitis, Henoch-Schönlein purpura, Polyarteritis nodosa, Takayasu arteritis, Giant cell arteritis), EMA algorythm (Microscopic polyangiitis) or 2012 revised Chapell Hill nomenclature criteria (Variable Vessel Vasculitis, Single-Organ Vasculitis). ANCA-Associated Vasculitis (AAV) was used to define Small Vessel Vasculitis (SVV) not fulfilling either ACR 1990 criteria or EMA algorythm.

Results: A total of 56 patients were included. Baseline characteristics of patients were as follows: female gender $75 \%$, mean age $56 \pm 15.7$ years, and median disease duration 3 (IQR 6) years. Most of patients (73\%) were diagnosed

Table 1. Demographic and immunologic characteristics.

\begin{tabular}{|l|c|}
\hline Female gender, $n$ [\%] & $42[75]$ \\
\hline Mean age at onset, years & $51 \pm 16.7$ \\
\hline Mean age at diagnosis, years & $53 \pm 15.9$ \\
\hline Polyautoimmunity* & $7[13]$ \\
Multiple Autoimmune Syndrome * & $6[9]$ \\
\hline AAV ANCA distribution & $\mathrm{n}[\%]$ \\
\hline C-ANCA/PR3 & $12[40]$ \\
P-ANCA/MPO & $11[37]$ \\
Both ANCA & $3[10]$ \\
No data & $4[13]$ \\
\hline Type of vasculitis & $\mathrm{n}[\%]$ \\
\hline Small Vessel & $31[55]$ \\
AAV & $19[34]$ \\
Granulomatosis with polyangiitis & $8[14]$ \\
Eosinophilic granulomatosis with & $2[4]$ \\
polyangiitis & \\
Microscopic polyangiitis & $1[2]$ \\
Henoch-Schönlein purpura & $1[2]$ \\
\hline Medium Vessel & $4[7]$ \\
Polyarteritis nodosa & $4[7]$ \\
\hline Large Vessel & $13[23]$ \\
Takayasu arteritis & $5[9]$ \\
Giant cell arteritis & $8[14]$ \\
\hline Other & $8[14]$ \\
Behçet & $3[5]$ \\
Aortitis & $1[2]$ \\
Skin-limited & $3[5]$ \\
Cogan syndrome & $1[2]$ \\
\hline The presence of more than one autoimmune disease in a single \\
patient. Distribution of autoimmune comorbidities were as follows: \\
antiphospholipid syndrome, systemic lupus erythematosus-SLE like $(3$ \\
each); autoimmune hepatitis, autoimmune \\
myasthenia gravis, pyoderma gangrenosum, rheumatoid arthritis, \\
Sjögren's syndrome, and vitiligo (1 each). \\
Multiple Autoimmune Syndrome: The presence of three or more \\
well-characterized autoimmune diseases in the same patient. \\
* A case of TAK with positive C-ANCA was excluded. \\
\hline
\end{tabular}

at first year of disease onset, and fulfilled international classification criteria (63\%). SVV was the most frequent phenotype (55\%), and C-ANCA/anti-PR3 were the most frequently identified auto-antibodies (40\%). Musculoskeletal manifestations (arthralgia, arthritis, and myalgia), mucocutaneous disorders (including vasculitic/necrotic lesions, mucosal ulcers, and purpura), neurological compromise (peripheral and central), and renal involvement (acute renal failure, glomerulonephritis, and lung-kidney syndrome) were the most frequently reported onset symptoms in $29 \%, 29 \%, 23 \%$, and $21 \%$, respectively. Interestingly, most of patients did not develop organic compromise other than the onset manifestation form. Thirteen percent of patients fulfilled polyautoimmunity criteria and $9 \%$ presented with multiple autoimmune syndrome. Antiphospholipid syndrome was the most common associated autoimmune disease described. Corticosteroids were the most common treatment used in $93 \%$ of patients, followed by azathioprine in $57 \%$, cyclophosphamide, methotrexate, and rituximab in $29 \%$, and antimalarials in $27 \%$ (Tab.1). No deaths occurred during follow-up.

Conclusions: Vasculitides are conditions with several subphenotypes, being ANCA-associated the most frequently reported. Onset symptoms seem to be the main drivers of disease evolution. Appropriate and prompt diagnosis is critical to enable timely intervention, aimed to prevent end organ damage and reduce morbidity in these patients. Controlling disease activity and preventing progression is the milestone of treatment. Characterization of Latin America population is pivotal to raise awareness of health-care workers, and policy makers.

\section{References:}

[1] Khosla A, et al. Radiol Clin North Am. 2016;54(3):613-28.

[2] Iglesias Gamarra A, et al. Med Sci Monit. 2010;16(3):RA58-72.

Disclosure of Interest: None declared

DOI: 10.1136/annrheumdis-2017-eular.6766

\section{AB0571 BIRMINGHAM VASCULITIS ACTIVITY SCORE MORE THAN 9.5 AT DIAGNOSIS IS AN INDEPENDENT PREDICTOR OF REFRACTORY DISEASE OF GRANULOMATOSIS WITH POLYANGITIS}

J. You, H.J. Kim, S.-W. Lee. Division of Rheumatology, Department of Internal Medicine, Yonsei University College of Medicine, Seoul, Republic of Korea, Seoul, Korea, Republic Of

Background: Granulomatosis with polyangiitis (GPA), that is identical to what has been called Wegener's granulomatosis, is one of anti-neutrophil cytoplasmic antibody (ANCA)-associated vasculitides (AAV). GPA is characterized by necrotising granulomatous inflammation usually affecting small to medium vessels, and it often involves the upper and lower respiratory tracts and commonly provokes necrotising glomerulonephritis. It has been reported that the mortality rate in untreated patients increased up to $90 \%$ within 2 years after diagnosis, and the overall mortality rates were ranging from $12 \%$ to $44 \%$ within 4 to 10 years. The major causes of death are known as cardiovascular disease, adverse events of immunosuppressive agents and major organ involvement of GPA. If there might be predictors of relapse or refractory disease of GPA during the follow-up duration, they can help physicians to select the induction therapeutic regimens, decide the duration of the maintenance therapeutic regimens and adjust the follow-up interval in order to improve the disease course of GPA.

Objectives: We investigated whether clinical manifestations, anti-neutrophil cytoplasmic antibodies (ANCAs), Birmingham vasculitis activity score (BVAS) for granulomatosis with polyangiitis (GPA) and five factor score (FFS) at diagnosis can predict relapse or refractory disease in 30 histology-proven GPA patients with the follow-up duration $\geq 12$ weeks.

Methods: We reviewed the medical records of 30 GPA patients. We collected clinical data, ANCAs, BVAS for GPA, FFSs at diagnosis, and we compared variables between the two groups based on relapse or refractory disease. The optimal cut-offs were extrapolated. Multivariate logistic regression and Cox hazard model analyses were conducted to identify predictors of refractory disease.

Results: The mean age and follow-up duration of patients were 63.3 years old and 45.2 months. The mean initial BVAS for GPA, FFS (1996) and FFS (2009) were 5.4, 0.6 and 1.0. There were no significant predictors of relapse. The mean BVAS for GPA, FFS (1996) and FFS (2009) of patients with refractory disease were higher than those without $(p<0.05$ for all). Patients having BVAS for GPA $\geq 9.5$, FFS $(1996) \geq 2$ and FFS $(2009) \geq 2$ exhibited significantly enhanced risk of refractory disease than those having not (RR 23.0, RR 11.0, and RR 55.0, respectively), and low cumulative refractory disease free survival rates. Multivariate Cox hazard model analysis proved BVAS for GPA $>9.5$ was an independent predictor of refractory disease during the follow-up duration (OR 12.892).
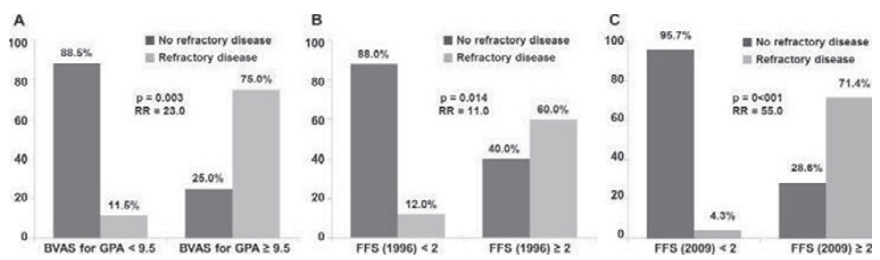

Conclusions: BVAS for GPA $\geq 9.5$ was an independent predictor of refractory disease during the follow-up duration $\geq 12$ weeks. 